\title{
Respuesta cardíaca de defensa y emocionalidad negativa en población normal
}

\author{
NiEVES FuENTES-SÁNCHEZ \\ al262229@uji.es \\ IRENE JAÉN \\ al119335@uji.es \\ BEATRIZ GARCÍA-CARRIÓN \\ al289008@uji.es \\ Eva CIFRE \\ cifre@uji.es \\ M. CARMEN PASTOR \\ mpastor@uji.es
}

\section{Resumen}

La Respuesta Cardíaca de Defensa (RCD) es un patrón dinámico de reactividad cardíaca en respuesta a un estímulo aversivo, intenso e inesperado que consiste en dos componentes sucesivos acelerativos y decelerativos, cuya significación psicológica se atribuye tanto a procesos atencionales como emocionales. De acuerdo con el modelo de la cascada defensiva propuesto por Lang (1995), el segundo componente acelerativo de la RCD puede interpretarse como un indicador de activación del sistema motivacional defensivo. Esta investigación tiene como objetivo analizar la relación entre la RCD y los niveles de ansiedad y depresión, como indicadores de emocionalidad negativa. La muestra experimental fue de 100 participantes, estudiantes de Psicología y Criminología de la Universitat Jaume I. La tarea experimental consistió en la presentación de un sonido intenso y aversivo $(500 \mathrm{~ms}, 105 \mathrm{~dB})$ tras un periodo de reposo de 6 minutos. El registro de los componentes acelerativos y decelerativos se realizó durante los $80 \mathrm{~s}$ posteriores a la presentación del estímulo acústico. Al finalizar la sesión experimental se administró una batería de cuestionarios, entre ellos el BDI y el STAI-R. Los análisis preliminares muestran una mayor reactividad cardíaca en los participantes con mayores puntuaciones en ansiedad y depresión. Estos datos sugieren que una mayor reactividad del sistema motivacional defensivo podría interpretarse como un factor de vulnerabilidad para desarrollar trastornos de ansiedad y depresión. Podemos concluir, por tanto, que la RCD parece ser un indicador fiable y válido de la hiperactividad del sistema motivacional defensivo en personas que presentan una mayor predisposición a reaccionar con sintomatología ansiosa o depresiva.

Palabras clave: Respuesta Cardíaca de Defensa, ansiedad, depresión, emocionalidad negativa, población normal 


\begin{abstract}
The Cardiac Defense Response (CDR) is a dynamic pattern of cardiac reactivity in response to an aversive, discrete, intense and unexpected stimulus consisting of two successive accelerative and decelerative components whose psychological significance is attributed to both attentional and emotional processes. According to the model of the defensive cascade proposed by Lang (1995), the second accelerative component of the CDR can be interpreted as an indicator of activation of the defensive motivational system. This study aims to analyze the relationship between CDR and levels of anxiety and depression as indicators of negative emotionality. The sample consisted of 100 participants, students of Psychology and Criminology, from Jaume I University. The experimental task consisted in the presentation of intense and aversive sound $(500 \mathrm{~ms}$, $105 \mathrm{~dB}$ ) after a 6-minute rest period. Accelerative and decelerative components were obtained during the $80 \mathrm{~s}$ after the presentation of the acoustic stimulus. At the end of the experimental session, a battery of questionnaires was administered, including BDI and STAI-R. Preliminary results show higher cardiac reactivity in participants with higher scores both on anxiety and depression. These data suggest that a greater reactivity of the defensive motivational system can be a factor of vulnerability to develop anxiety and depression disorders. We can conclude, therefore, that CDR may be interpreted as an index of the hyperactivity of the defensive motivational system in people with greater predisposition to react with anxious or depressive symptoms.
\end{abstract}

Key Words: Cardiac Defense Response, Anxiety, Depression, negative emotionality, normal population.

\title{
Introducción
}

El origen del concepto de defensa está relacionado con la idea de que los organismos reaccionan fisiológicamente ante la presencia de peligro o amenaza con el objetivo de protegerse a sí mismos del daño o la muerte. Entre las respuestas típicas de defensa en numerosas especies podemos encontrar el freezing, la reacción de sobresalto, el desmayo y la respuesta de lucha-huida. Todas estas reacciones pueden ser categorizadas en dos formas generales de defensa: inmovilidad y defensa activa (Vila, y cols., 2007).

La Respuesta Cardíaca de Defensa (RCD) constituye un patrón de reactividad cardíaca observado después de la presentación de un estímulo intenso y aversivo (mayoritariamente auditivo o electrocutáneo). Este patrón cardíaco implica dos componentes acelerativos (una aceleración de corta latencia y una segunda aceleración de larga latencia), y dos componentes decelerativos -que aparecen secuencialmente durante los 80 segundos después de la presentación del estímulo aversivo. Los mecanismos fisiológicos de este patrón de respuesta incluyen influencias simpáticas y parasimpáticas. La primera aceleración/deceleración es controlada por influencias parasimpáticas (i.e., inhibición durante la primera aceleración y activación durante la subsecuente deceleración). Por su parte, la segunda aceleración/deceleración es controlada dualmente por las ramas simpáticas y parasimpáticas que actúan de manera recíproca, observándose activación simpática -acompañada por inhibición parasimpática- durante la segunda aceleración, e inhibición simpática -acompañada por activación parasimpáticadurante la segunda deceleración (Vila, y cols., 2007). 


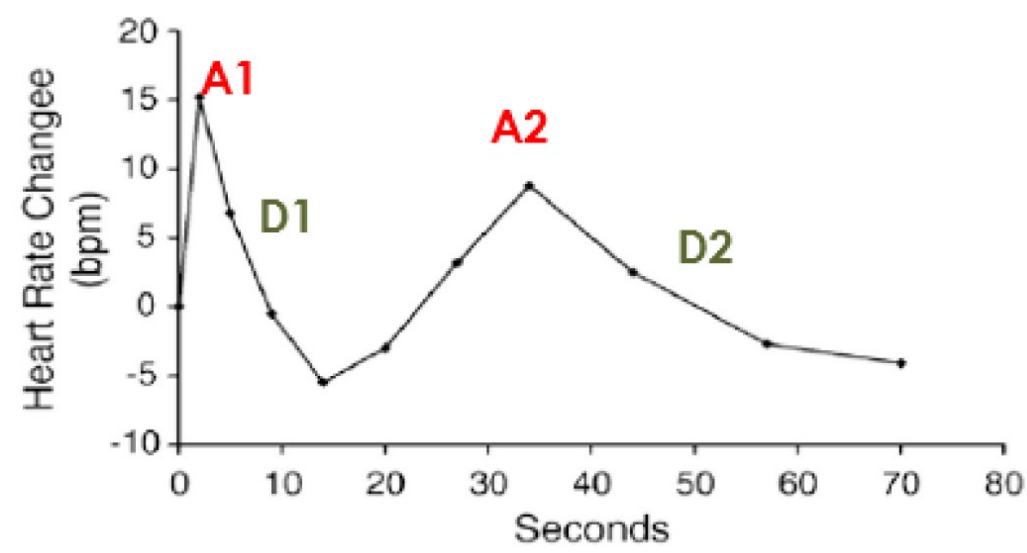

Figura 1. Componentes acelerativos y decelerativos del patrón de RCD (Adaptado de Vila, y cols., 2008)

Existen dos aproximaciones tradicionales que intentan explicar esta respuesta autonómica. La aproximación cognitiva distingue entre el reflejo de orientación y el reflejo defensivo, asumiendo que los cambios cardíacos en respuesta a los estímulos del ambiente reflejan mecanismos atencionales y perceptivos. Desde esta aproximación, el reflejo de orientación (una deceleración de la tasa cardíaca) facilita la atención hacia el estímulo, mientras que la respuesta defensiva (una aceleración de la tasa cardíaca) reduce la atención hacia el estímulo como una forma de protección contra el estímulo amenazante (Vila, y cols., 2007)). Por su parte, la aproximación motivacional asume que los cambios cardíacos en respuesta a los estímulos ambientales reflejan mecanismos metabólicos con el objetivo de proporcionar al organismo la energía necesaria para actuar. Cuando se requiere una respuesta activa, tanto psicológica como fisiológica, la reacción se acompañará de aceleración cardíaca, pero si es más apropiado responder con una conducta pasiva el organismo presentará una clara deceleración cardíaca (Vila, y cols., 2007).

Sin embargo, en los últimos años han ido surgiendo nuevas investigaciones que están facilitando la integración de ambas tradiciones psicológicas clásicas dentro de un mismo modelo teórico atencional-motivacional de defensa cardíaca (Vila, y cols., 2009). Concretamente, nos referimos al modelo de la cascada defensiva, desde el cual se entiende que las reacciones defensivas siguen un proceso secuencial con fases iniciales atencionales dirigidas a la detección y análisis de la posible amenaza, seguidas de fases posteriores en las que predominan los factores motivacionales dirigidos a facilitar las acciones defensivas de lucha o huida (Blanchard y Blanchard, 1989; Fanselow, 1994; Lang, Davis y Öhman, 2000).

Desde esta perspectiva, la respuesta cardíaca de defensa -al igual que sucede con otras respuestas defensivas, como el reflejo de sobresalto- está modulada tanto por factores atencionales como emocionales. Así, los componentes acelerativos y decelerativos parecen reflejar la sucesión de dos fases: (1) una primera fase defensiva atencional, dirigida a interrumpir la actividad y analizar el peligro potencial, y (2) una fase defensiva motivacional, dirigida a preparar al organismo para la defensa activa. De esta forma, el patrón de respuesta cardíaca de defensa representa una transición desde la atención (orientación) a la acción (respuesta abierta). La primera aceleración/deceleración implica la interrupción de la actividad y el aumento de la atención hacia los estímulos externos, mientras que la segunda aceleración/deceleración prepara al organismo para la defensa y permite su recuperación en el caso de que el peligro no sea real (Vila, y cols., 2007). 


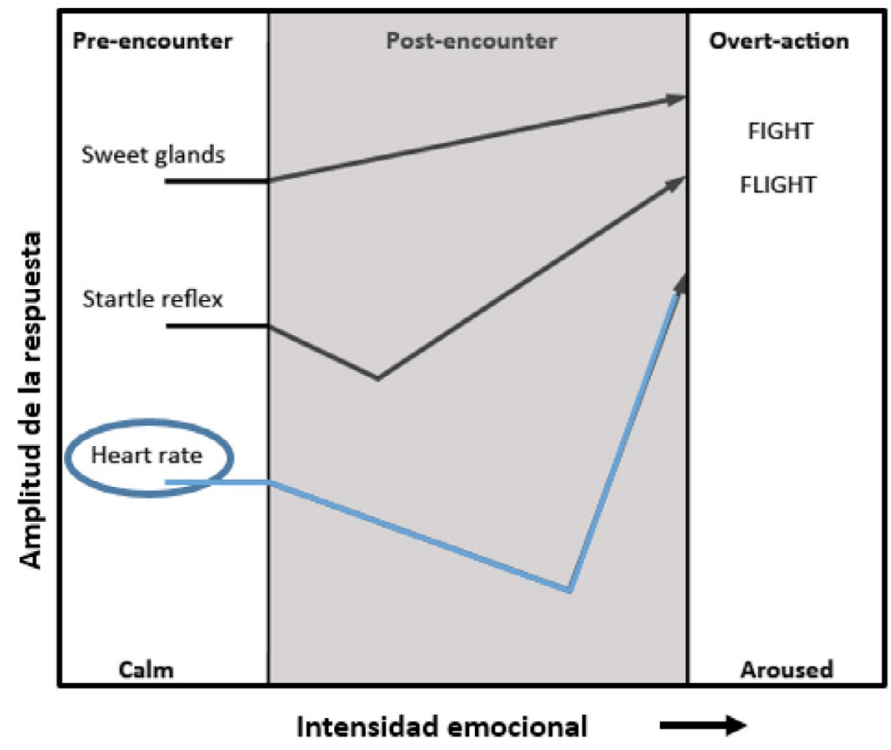

Figura 2. Modelo de la cascada defensiva (Adaptado de Bradley y Lang, 2000)

Debido a su significado emocional, el presente estudio se centra en el segundo componente acelerativo, el cual tiene un pico de máxima respuesta entre los 20 y 45 segundos posteriores a la presentación del estímulo intenso y aversivo que provoca la reacción defensiva. Esta segunda aceleración cardíaca refleja la movilización de los recursos del organismo para dar una respuesta de afrontamiento y se ha interpretado como un buen indicador de la activación del sistema motivacional defensivo (López, y cols., 2016). De relevancia para nuestro trabajo, los resultados de la investigación previa han mostrado importantes diferencias individuales en el patrón de respuesta cardíaca a partir de dicho componente acelerativo. Más concretamente, teniendo en cuenta la presencia o ausencia de esta segunda aceleración, podemos clasificar a las personas como aceleradoras o deceleradoras. Mientras que las «aceleradoras» se caracterizan por presentar una clara aceleración cardíaca con una mayor latencia, las «deceleradoras» presentan una marcada deceleración (Pérez Marfil, Fernández-Santaella, León García, Turpin y Vila, 1999).

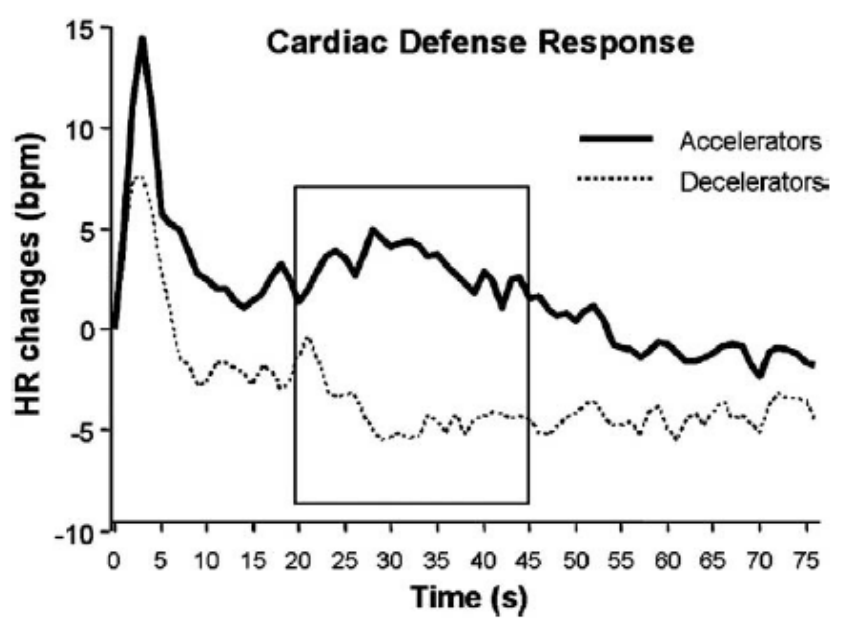

Figura 3. Patrones de RCD para aceleradores y deceleradores en el test de reactividad cardíaca (Tomado de López-Penades, y cols., 2008) 
Estas diferencias en el patrón de la respuesta han sido asociadas con factores tanto biológicos como psicológicos, como pueden ser el ciclo menstrual, el género, los rasgos de personalidad, la preocupación excesiva o la ansiedad patológica. En concreto, se han encontrado patrones marcadamente acelerativos en hombres comparado con mujeres, en mujeres fóbicas durante la fase premenstrual vs. la fase inter-menstrual, en personas con altas puntuaciones en preocupación e inestabilidad emocional, así como en pacientes con trastornos de ansiedad (Vila, y cols., 2009). Concretamente, los datos indican que tanto las personas con elevadas puntuaciones en preocupación como los pacientes con ansiedad generalizada tienden a mostrar un patrón acelerativo prolongado (con ausencia del primer componente decelerativo). Sin embargo, las personas con una elevada ansiedad subclínica y los pacientes con fobias específicas tienden a mostrar el patrón típico de RCD con ambos componentes de manera secuencial (acelerativo/desacelerativo) (Vila, y cols., 2009; Vila, y cols., 2003).

El presente trabajo de investigación pretende explorar si se cumplen dos hipótesis formuladas a partir de la revisión de la literatura experimental previa. En primer lugar, se espera replicar el patrón de RCD (con sus dos componentes) en una muestra de estudiantes representativa de la población normal y, en segundo lugar, se pretende explorar si existen diferencias entre las personas aceleradoras y deceleradoras (clasificadas en función de la segunda aceleración de la RCD) en su emocionalidad negativa, evaluada a partir de varios cuestionarios de afecto. En relación con el segundo objetivo y, de acuerdo con la literatura, esperamos encontrar mayores puntuaciones en los distintos instrumentos de autoinforme para el grupo de aceleradores comparado con el grupo de deceleradores.

\section{Método}

\section{Participantes}

En este estudio participaron voluntariamente 100 estudiantes del grado de Psicología y Criminología (30 varones y 70 mujeres) de la Universitat Jaume I. Los participantes tenían una media de edad de 21.15 años (DT = 2.64), sin observarse diferencias de edad significativas entre la muestra de varones (Media $=21.73$; DT $=2.54$ ) y la de mujeres (Media $=20.87$; $\mathrm{DT}=$ 2.66). Previamente se comprobó que ningún participante tuviera problemas auditivos o cardiovasculares, ni tampoco se encontrara bajo tratamiento farmacológico o psiquiátrico. El presente estudio forma parte de un proyecto de investigación más amplio aprobado por el Comité Deontológico de la Universidad Jaume I.

\section{Materiales}

Inventario de la Depresión de Beck [BDI-II] (Beck, y cols., 1996): cuestionario formado por 21 ítems relacionados con síntomas depresivos (sentimientos de culpa, sentimientos físicos relacionados con la depresión, etc.) para evaluar la severidad de la depresión. Todos los ítems tienen como escala de respuesta 0,1, 2, 3 -excepto dos ítems (el 16 y 18), cuya escala de respuesta es $0,1,1,2,2,3,3$. Un ejemplo de ítem es el siguiente: «Me siento triste gran parte del tiempo».

Cuestionario de Regulación Emocional [ERQ] (Cabello, y cols., 2013): cuestionario de 10 ítems que evalúa dos estrategias específicas de regulación emocional, reevaluación cognitiva (6 ítems; "Cuando quiero incrementar mis emociones positivas (p.ej. alegría, diversión), cambio el tema sobre el que estoy pensando») y supresión emocional (4 ítems; "Cuando estoy 
sintiendo emociones positivas, tengo cuidado de no expresarlas»). Los ítems presentan una escala tipo Likert de 5 puntos (totalmente en desacuerdo; en desacuerdo; neutral; de acuerdo; totalmente de acuerdo).

Cuestionario de Ansiedad-Estado [STAI-E] (Spielberger, y cols., 1970; Spielberger, y cols., 1982): cuestionario de 20 ítems que evalúa la ansiedad estado, es decir, un estado emocional transitorio. Los ítems presentan una escala tipo Likert de 4 puntos (nada; algo; bastante; mucho). EL STAI-E tiene en cuenta una serie de síntomas en el momento en el que se administra el cuestionario. "Me siento descansado» es un ejemplo de ítem.

Cuestionario de Ansiedad-Rasgo [STAI-R] (Spielberger, y cols., 1970; Spielberger, y cols., 1982): cuestionario de 20 ítems que evalúa la ansiedad rasgo, es decir, componentes cognitivos y somáticos de la ansiedad como un rasgo general de la personalidad. Los ítems presentan una escala escala tipo Likert de 4 puntos (nada; algo; bastante; mucho). "Soy una persona tranquila, serena y sosegada» es un ejemplo de ítem.

Escala PANAS de afecto positivo y negativo (Watson, y cols., 1988): escala que consta de 20 ítems y evalúa afecto positivo y afecto negativo. Cada ítem presenta una escala de 5 puntos (muy ligeramente o nada; un poco; moderadamente; bastante; mucho). "Preocupado», «Fuerte», «Nervioso», «Activo», etc. son algunos ejemplos de ítems.

\section{Adquisición y reducción de datos fisiológicos}

Para la adquisición y el análisis de las distintas señales fisiológicas se utilizó un sistema de registro Biopac MP36 y el software Acqknowledge 4.1. La tasa cardíaca fue registrada mediante sensores de tamaño estándar ( $8 \mathrm{~mm}$ de diámetro) colocados según la derivación II: dos electrodos activos -en la muñeca derecha y en el tobillo izquierdo- y un electrodo de tierra en el tobillo derecho. Previamente se limpiaron las zonas descritas frotando con un algodón impregnado en alcohol. Los valores de tasa cardíaca se expresaron como puntuaciones de cambio respecto a la media de los 15 segundos previos a la presentación del estímulo auditivo (línea de base).Con ello, se obtuvieron 80 valores (correspondientes a los 80 segundos tras la aparición del estímulo auditivo). Para facilitar el análisis estadístico, estos 80 valores de tasa cardíaca fueron reducidos a 10 valores, correspondientes a las medianas de los 10 intervalos siguientes: 2 intervalos de 3 segundos, 2 intervalos de 5 segundos, 3 intervalos de 7 segundos, y 3 intervalos de 13 segundos.

\section{Procedimiento}

El estudio se llevó a cabo en el laboratorio del grupo MPAGER situado en la Facultad de Ciencias Humanas y Sociales de la Universitat Jaume I. Unos días antes del experimento, se enviaba a los/las participantes un correo electrónico donde se les indicaba el día y hora a la que estaban citados, así como unas recomendaciones que debían seguir antes de venir al experimento. Una vez en el laboratorio, debían firmar el consentimiento informado donde se les explicaba en qué iba a consistir la sesión experimental. A continuación, se limpiaban las zonas donde se colocaban los sensores y se procedía a leer las instrucciones de la tarea en las que se indicaba que debían estar en reposo (pero no se les avisaba de la presentación del estímulo aversivo). Todos los participantes realizaron un test de reactividad fisiológica para provocar la Respuesta Cardíaca de Defensa (Ver Figura 4). Esta tarea consistió en la presentación de un sonido intenso y aversivo (ruido blanco de 500 ms de duración, 105 dB y rise time instantáneo), administrado biauralmente a través de auriculares (Sennheiser HD 205), tras un 
periodo de reposo de 6 minutos. Después del sonido intenso hubo un periodo de registro de 80 segundos adicionales. Tras finalizar la tarea, se administraron diferentes cuestionarios de personalidad (BDI-II, ERQ, STAI-R, STAI-E, STAI-R y PANAS, en este orden), y se despedía a los participantes explicando brevemente el objetivo del estudio.
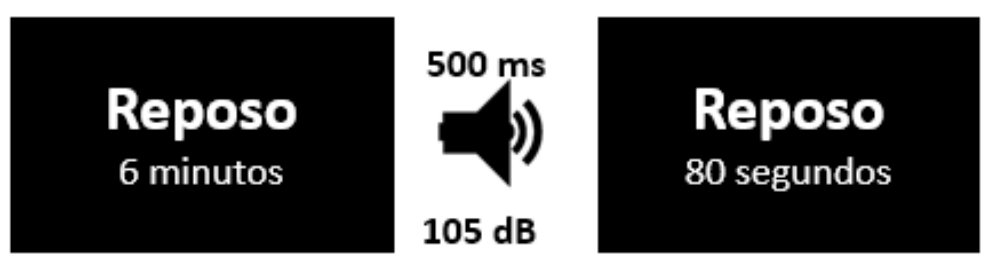

Figura 4. Test de reactividad cardíaca utilizado para provocar la Respuesta Cardíaca de Defensa (RCD)

\section{Análisis estadístico}

Para clasificar a la muestra experimental en dos grupos (aceleradores vs. deceleradores) se llevó a cabo un análisis de Cluster jerárquico (método de Ward y datos estandarizados) a partir del valor obtenido en la Mediana 7 (correspondiente a la segunda aceleración). A continuación, para cada uno de los cuestionarios por separado (BDI-II, ERQ, STAI-R, STAI-E, STAI-R y PANAS), se realizaron Análisis de Varianza de medidas repetidas (ANOVA) con el factor Grupo (Aceleradores vs. Deceleradores). Se fijó el nivel de significación de $p<.05$ usando la corrección Greenhouse-Geisser. Los análisis estadísticos se llevaron a cabo mediante el software JMP 5.0.1.

\section{Resultados}

\section{Patrón de Respuesta Cardíaca de Defensa (RCD)}

El análisis de Cluster realizado mostró la existencia de dos clusters que diferían significativamente en sus puntuaciones en el componente acelerativo utilizado para clasificarlos $[F$ $(1,98)=3.39, p<0.0002)$.

A partir de este análisis, la muestra experimental se clasificó en dos grupos: aceleradores $(N=49)$-con un patrón de reactividad cardíaca marcadamente acelerador-, y deceleradores $(\mathrm{N}=51)$-caracterizados por un patrón cardíaco básicamente decelerativo a lo largo de los 80 segundos posteriores a la presentación del sonido aversivo y, por tanto, por mostrar ausencia del segundo componente acelerativo entre 20 y 40 segundos. 


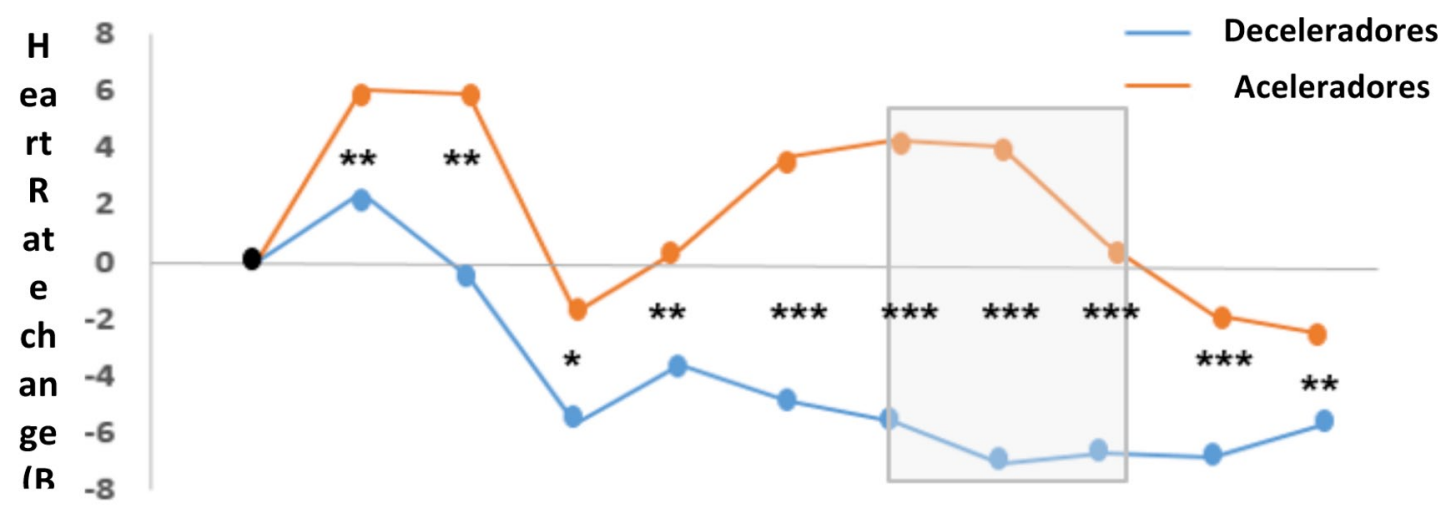

LB Md1 Md2 Md3 Md 4 Md 5 Md 6 Md 7 Md 8 Md 9 Md 10

Figura 5. Patrones de RCD en el test de reactividad cardíaca para aceleradores y deceleradores

\section{Respuesta Cardíaca de Defensa (RCD) y Emocionalidad negativa}

Los ANOVAs realizados mostraron la ausencia de diferencias estadísticamente significativas entre los dos grupos (aceleradores vs. deceleradores) para los distintos instrumentos de autoinforme administrados al finalizar el experimento ( $F s<1)$.

No obstante, tal y como se esperaba, se observó una tendencia congruente en la dirección de las diferencias al comparar las medias obtenidas en ambos grupos experimentales, tanto para el BDI como para el STAI (subescalas de Ansiedad Estado y Rasgo). Más concretamente, las personas aceleradoras puntuaron más alto tanto en depresión $[F(1,98)=0.11, p$ $<0.64)$ ] como en ansiedad estado $[F(1,98)=0.23, p<0.63)]$ y en ansiedad rasgo $[F(1,98)=$ $0.41, p<0.55]$, en comparación con las personas deceleradoras (ver Figura 6).

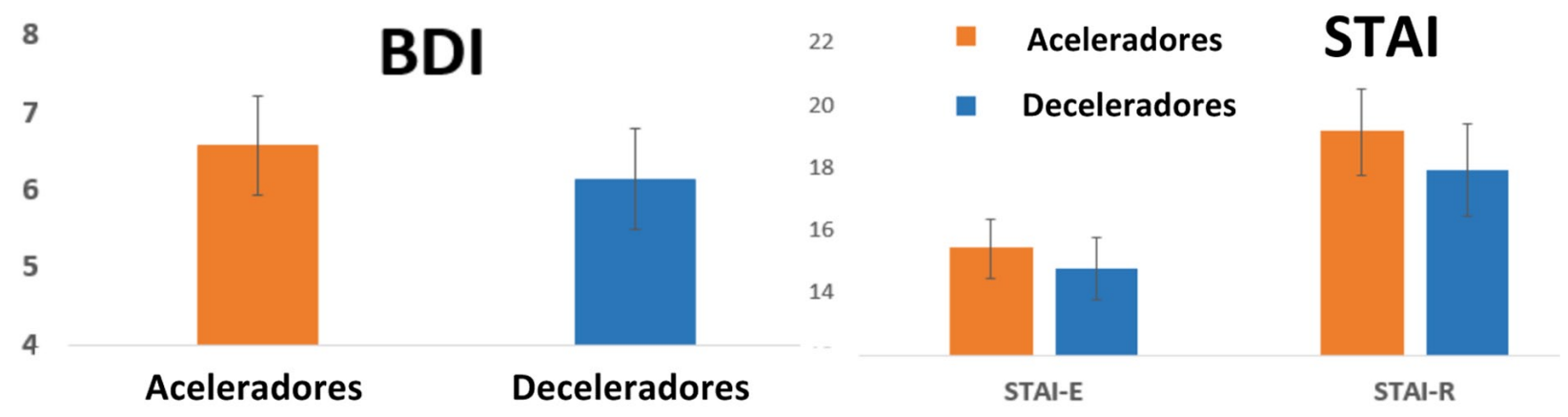

Figura 6. Medias en los cuestionarios BDI y STAI para los dos clusters (deceleradores y aceleradores)

Además, tampoco se obtuvieron diferencias estadísticamente significativas entre aceleradores y deceleradores en las puntuaciones en las escalas de Reevaluación Cognitiva [ $F$ $(1,98)=0.26, p<0.61)$ y Supresión $[F(1,98)=0.30, p<0.59]$ del ERQ (ver Figura 7$)$. 


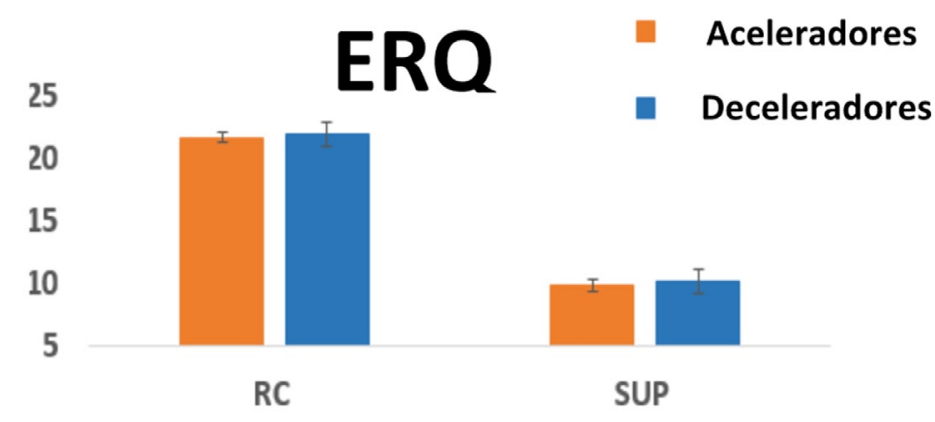

Figura 7. Medias en el cuestionario ERQ para las estrategias de Supresión (SUP) y Revaluación Cognitiva (RC), para los dos clusters (deceleradores y aceleradores)

Finalmente, no se observaron diferencias estadísticamente significativas entre ambos grupos en las puntuaciones del PANAS para las escalas de afecto negativo $[F(1,98)=0.57$, $p<0.45]$ y afecto positivo $[F(1,98)=0.12, p<0.72]$ (Ver Figura 8).

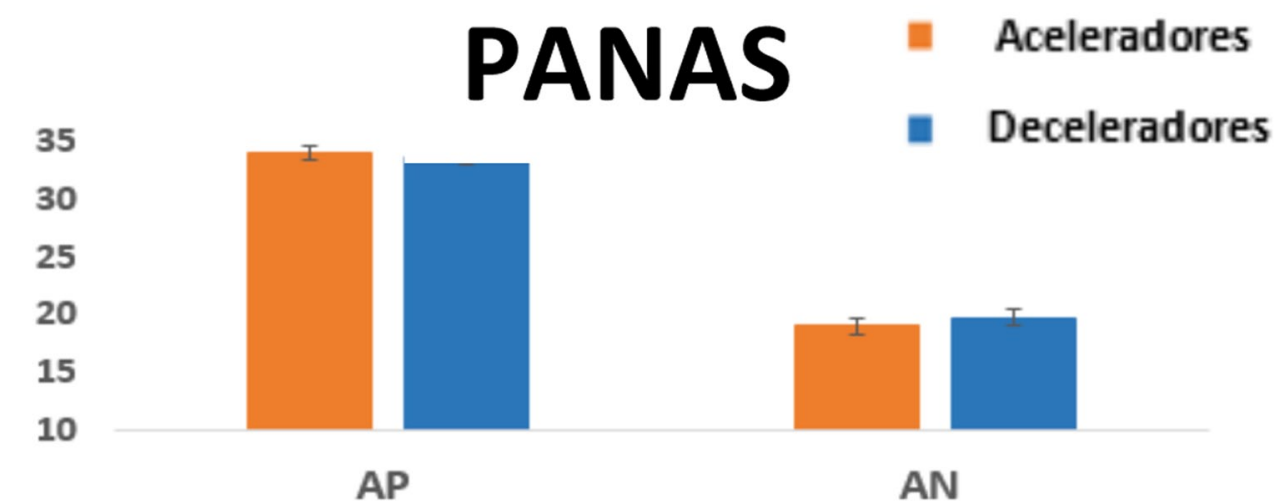

Figura 8. Medias en el cuestionario PANAS para las escalas de Afecto Negativo y Afecto Positivo, para los dos clusters (deceleradores y aceleradores).

\section{Discusión y Conclusiones}

En general, los datos del presente estudio han permitido replicar el patrón de RCD en una muestra de estudiantes, en el que se observan claramente dos componentes acelerativos y decelerativos de manera secuencial, de acuerdo con los resultados de trabajos previos (Vila, y cols., 2007; Vila, y cols., 2003). Asimismo, se ha conseguido replicar la existencia de dos clusters diferenciados a partir del segundo componente acelerativo de la RCD (Eves y Gruzelier, 1984; Fernández y Vila, 1989, López-Penades, y cols., 2009). En esta línea, los resultados muestran un grupo de personas claramente aceleradoras y un grupo de personas deceleradoras, que difieren significativamente en la segunda aceleración cardíaca. Esta segunda aceleración representa la preparación para la defensa activa en caso de que ocurriera un peligro real y, por tanto, refleja la activación del sistema motivacional defensivo. 
Por otro lado, cuando se analizan si existen diferencias entre aceleradores y deceleradores en diferentes instrumentos de autoinforme utilizados para evaluar emocionalidad negativa no se encuentran diferencias significativas entre ambos clusters. Sin embargo, al analizar el sentido de las diferencias, sí se observa un patrón acorde con la literatura previa (López, y cols., 2016; Pérez, Fernández, Vila, y Turpin, 2000). En concreto, las personas con mayor reactividad cardíaca presentaron mayores puntuaciones en las escalas de ansiedad y depresión. Estos datos muestran cómo el patrón de RCD puede verse alterado por el estado emocional de la persona. Cuando la persona se encuentra en un estado emocional negativo se produce una segunda aceleración pronunciada y una ausencia (o una menor) segunda deceleración. Esto sugiere, siguiendo el modelo de la cascada defensiva, el inicio de la fase motivacional -lo cual se acompaña de una aceleración cardíaca que facilitaría, por tanto, la reacción defensiva.

No obstante, aunque estos datos van en la línea de la literatura previa, en nuestro estudio no se encuentran diferencias significativas entre ambos grupos de participantes a partir de los cuestionarios utilizados para evaluar afecto. Este resultado podría explicarse por el reducido tamaño de la muestra experimental que podría resultar inadecuado cuando se utiliza una batería de autoinformes. A esta primera limitación se suma el hecho de que se trata de una muestra no clínica, observándose claramente que las puntuaciones obtenidas en las distintas escalas están dentro de la normalidad. En consecuencia, los valores medios obtenidos en los cuestionarios fueron muy similares entre sí (con una desviación típica muy baja), dificultando de este modo la aparición de diferencias estadísticamente significativas entre aceleradores y deceleradores. Por último, cabe mencionar una limitación referida al propio test de reactividad cardíaca utilizado en el presente estudio, ya que la tarea original cuenta con 10 minutos de reposo antes de la presentación del sonido intenso (Vila, y cols., 2007; Vila, y cols., 2003). Sin embargo, en el presente trabajo se tomaron únicamente 6 minutos de reposo como línea de base, de acuerdo con estudios previos (López, y cols., 2009), para reducir la duración de la tarea dado que iba seguida de otro experimento psicofisiológico. En consecuencia, cabe explorar si el tiempo de reposo antes de la aparición del estímulo aversivo utilizado para provocar la respuesta cardíaca de defensa puede ser un factor a tener en cuenta en futuros estudios.

Aunque la investigación sobre las reacciones defensivas ha sido muy extensa en los últimos años, aún se desconocen numerosos aspectos básicos referentes a dichas respuestas, por lo que es necesario continuar el estudio de estos procesos. Por ejemplo, una posible línea futura es el estudio de los mecanismos corticales y vegetativos de los diferentes patrones acelerativos y decelerativos, así como su papel en los estilos defensivos activo/pasivo en los distintos trastornos emocionales.

\section{Referències bibliogràfiques}

Bradley, M.M., y Lang, P.J. (2000). Affective reactions to acoustic stimuli. Psychophysiology, 37, 204-215.

Eves, F.F. y Gruzelier, J.M. (1984). Individual differences in the cardiac response to high intensity auditory stimulation. Psychophysiology, 21, 342-352.

Fernández, M.C. y Vila, J. (1989). La respuesta cardíaca de defensa en humanos: diferencias sexuales e individuales. Boletín de Psicología, 22, 59-89.

Lang, P.J. (1995). The emotion probe: Studies of motivation and attention. American Psychologist, 50, 372-385. 
Lang, P., Bradley, M. M., y Cuthbert, B.N. (1997). Motivated attention: Affectm activation, and action. In P. Langm R.F. Simons, y M.Balaban (Eds.), Attention and orienting: Sensory and motivational processes (pp. 97-136). Hillsdale, NJ: Erlbaum.

López, R., Poy, R., Pastor, M.C., Segarra, P., y Moltó, J. (2009). Cardiac defense response as a predictor of fear learning. International Journal of Psychophysiology, 74, 229-235.

López, R., Poy, R., Segarra, P., Esteller, À., Fonfría, A., Ribes, P., Ventura, C., y Moltó, J. (2016). Gender-specific effects of trait anxiety on the cardiac defense response. Personality and Individual Differences, 96, 243-247.

Pérez Marfil, M.N., Fernández Santaella, M.C., León García, A., Turpin, G., y Vila Castellar, J. (1999). Individual differences associated with cardiac defence response: psychophysiological and personality variables. Psychology in Spain, 3, 54-62.

Pérez, M.N., Fernández, M.C., Vila, J., y Turpin, G. (2000). Cognitive and emotional modulation of the cardiac defense response in humans. Psychophysiology, 37, 275-282.

Vila, J., Fernández, M.C., Pegalajar, J., Vera, M.N., Robles, H., Pérez, N., Sánchez, M.B., Ramírez, I., y Ruiz-Padial, E. (2003). A new look at Cardiac Defense: Attention or Emotion?. The Spanish Journal of Psychology, 6, 60-78.

Vila, J., Guerra, P., Muñoz, M.A., Vico, C., Viedma-del Jesús, M.I., Delgado, L.C., Perakakis, P., Kley, E., Mata, J.L., y Rodriguez, S. (2007). Cardiac defense : From attention to action. International Journal of Psychophysiology, 66, 169-182.

Vila, J., Guerra, P., Muñoz, M.A., Perakakis, P., Delgado, L.C., Figueroa, M., y Mohamed, S. (2009). La dinámica del miedo: la cascada defensiva. Escritos de Psicología, 3(1), $37-42$. 